\title{
Thymidylate Synthase Polymorphisms and Risk of Lung Cancer among the Jordanian Population: a Case Control Study
}

\author{
Wiam Al Qasem, Al-Motassem Yousef*, Mohammad Yousef, Ihab Manasreh
}

\begin{abstract}
Background: Thymidylate synthase (TS) catalyzes the methylation of deoxyuridylate to deoxythymidylate and is involved in DNA methylation, synthesis and repair. Two common polymorphisms have been reported, tandem repeats in the promoter-enhancer region (TSER), and 6bp ins/del in the 5'UTR, that are implicated in a number of human diseases, including cancer. The association between the two polymorphisms in risk for lung cancer (LC) was here investigated in the Jordanian population. Materials and Methods: An age, gender, and smoking-matched case-control study involving 84 lung cancer cases and 71 controls was conducted. The polymerase chain reaction/restriction fragment length polymorphism (PCR-RFLP) technique was used to detect the polymorphism of interest. Results: Individuals bearing the ins/ins genotype were 2.5 times more likely to have lung cancer [ $(95 \% \mathrm{CI}: 0.98-6.37), \mathrm{p}=\mathbf{0 . 0 5 1}$ ]. Individuals who were less than or equal to 57 years and carrying ins/ins genotype were 4.6 times more susceptible to lung cancer [OR<57 vs $>57$ years: 4.6 (95\% CI: 0.93-22.5), $\mathrm{p}=0.059)]$. Genotypes and alleles of TSER were distributed similarly between cases and controls. Weak linkage disequilibrium existed between the two loci of interest (Lewontin's coefficient [D']) (LC: D'=0.03, r2: 0. 001, p= 0.8; Controls: D' $=0.29, \mathrm{r} 2: 0.08, p=0.02)$. Carriers of the " 3 tandem repeats_insertion" haplotype (3R_ins) were 2 times more likely to have lung cancer [ $2(95 \% \mathrm{CI}: 1.13-3.48), p=0.061]$. Conclusions: Genetic polymorphism of TS at 3 UTR and its haplotype analysis may modulate the risk of lung cancer in Jordanians. The 6bp ins/del polymorphism of TS at $3^{\prime}$ UTR is more informative than TSER polymorphism in predicting increased risk.
\end{abstract}

Keywords: Jordan - TS - polymorphism - lung cancer - 3 `UTR - 6 bp ins/del

Asian Pac J Cancer Prev, 16 (18), 8287-8292

\section{Introduction}

Lung cancer is a heterogeneous, complex, and challenging disease to treat (Hensing et al., 2014) and is the leading cause of cancer-related death worldwide $(\mathrm{Wu}$ et al., 2014). In Jordan lung cancer is firmly proved as the second most common cancer in men, and the eighth most common cancer in women (Tarawneh et al., 2010) This fact is particularly bothering because lung cancer is one of the most preventable malignancy (Islami et al., 2015). Tobacco consumption is the primary cause of lung cancer. Moreover, one in 5 women and one in 12 men diagnosed with lung cancer have never smoked, suggesting that other risk factors are implicated in lung cancer development (pulmonary fibrosis, occupational and environmental exposure, genetics....etc.) (Horn et al., 2012). Cells may predispose to carcinogenesis if genetic alterations occur in any critical regulatory pathway; the main critical regulatory pathways include regulations of xenobiotic metabolism, control of genomic stability, including deoxyribonucleic acid (DNA) repair mechanisms, cellcycle checkpoints, apoptosis, and control of growth and inflammatory factors (Wu et al., 2004). Thymidylate synthase is one of the enzymes involved in one carbon- unit metabolism and thus DNA synthesis and repair. Thymidylate synthase (ts) catalyzes the methylation of deoxyuridylate to deoxythymidylate utilizing 5,10methylene-THF as a cofactor (Longley et al., 2003; Lv et al., 2013). ts has three polymorphisms that may affect ts expression; two functionally important polymorphisms; one is a promoter-enhancer region (TSER), containing 2 or 3 tandem repeats of 28 bp units (2R or 3R) (rs34743033; NC_000018.10: 657645-657731) and known to play a role in the transcriptional autoregulation mechanism (Horie et al., 1995). The second, common polymorphism is 6bp ins/ del-type polymorphism at ts1494del611 (rs151264360; NC_000018.10: 673444) localized on the 3' UTR, a region conceived to affect ts transcript stability and ts mRNA levels (Horie et al., 1995). Less functionally important polymorphism is a single nucleotide polymorphism (SNP) $\mathrm{C}>\mathrm{G}$ at the twelfth nucleotide of the second repeat of $3 \mathrm{R}$ allele (rs2853542; NC_000018.10: 657685) (Horie et al., 1995).

Significant association (linkage disequilibrium) between ts triple tandem repeat genotype (3R/3R) in TSER and del/del genotype in 3'UTR was found in rheumatoid arthritis Japanese patients (Kumagai et al., 2003), and Caucasian (Ulrich et al., 2002). Though a meaningful 
linkage disequilibrium was also detected between double tandem repeat $(2 \mathrm{R} / 2 \mathrm{R})$ genotypes and ins/ins genotype (Ulrich et al., 2002). This association was statistically significant in non-Hispanic Whites, Hispanic, and African American, but the association did not reach significance in Singapore Chinese population ( $\mathrm{p}$ value 0.15 ). Strong trend was found; $59 \%$ of all 3R/3R homozygotes were also homozygous for the $6 \mathrm{bp}$ deletion (Mandola et al., 2004). These findings are promising and provide some explanations of how an individual that is known to be homozygous for the $2 \mathrm{R}$ tandem repeat polymorphism having high ts gene expression, thus screening for both polymorphism may provide clear estimate for ts protein expression in vivo.

The aim of this study to ascertain if the two functionally important ts polymorphisms and their combined effect are associated with an altered risk of developing lung cancer among Jordanians.

\section{Materials and Methods}

\section{Clinical setting}

Lung cancer patients (cases) were recruited from the oncology clinic at Al- Basher Hospital $(\mathrm{ABH})$, and the Jordan University Hospital (JUH), Amman, Jordan after obtaining Institutional Review Boards (ABH:17421/IRB/ MBA; JUH: 49/2013/IRBJ). Sample collection started on 22th of January 2014 and finished on 1st of May 2014. Age, smoking status and gender matched healthy volunteers (control) were recruited from blood bank in JUH during September, 2014.

\section{ts Genotyping}

Four milliliters of venous blood were collected from patients and healthy subjects in K3EDTA coated tubes. The tubes were kept in icebox and DNA extraction was performed in the same day using Genomic Wizard DNA purification kit (Promega Corporation, USA) according to manufacturer's instructions. Amplification of the $6 \mathrm{bp}$ ins/del region was performed using the forward primer GTCTTTAGGGGTTGGGCTGG (NC_000018.10: 673031-673050) and the reverse primer AAAGCGTGGACGAATGCAGA (NC_000018.10: 673507-673488) to yield a 480-bp amplicon. All customized primers were synthesized by Princess Haya Biotechnology Centre at the King Abdullah University Hospital, Jordan University for Science and Technology (Irbid, Jordan). The PCR conditions for "6bp ins/del" (S 1000TM Thermal Cycler, Bio-Rad, USA) were: 5 minutes of initial denaturation at $95^{\circ} \mathrm{C}$, followed by 39 cycles of $95^{\circ} \mathrm{C}$ for 15 seconds, $60^{\circ} \mathrm{C}$ for 15 seconds, and $72^{\circ} \mathrm{C}$ for 20 seconds, with a final extension at $72^{\circ} \mathrm{C}$ for 5 minutes. The PCR products were further digested with Dra1 restriction enzyme (New England Biolab, USA). Resulting fragments were separated on $2 \%$ agarose gel electrophoresis and then visualized using RedSafe ${ }^{\mathrm{TM}}$ DNA staining (ChemBio, UK). The digested fragment sizes for the ts $6 \mathrm{bp}$ ins/del genotypes were: $480 \mathrm{bp}$ band for del/del, 480,415, 65bp bands for ins/del, and 415, $65 \mathrm{bp}$ bands for ins/ins.

The primers used to amplify the "TSER" polymorphism were: forward primer GTGGCTCCTGCGTTTCCCCC
(NC_000018.10: 657523-657542) and the reverse primer GGCTCCGAGCCGGCCACAGGCATGGCGCGG (NC_000018.10: 657764-657736). The PCR conditions for "TSER" were 5 minutes of initial denaturation at $94^{\circ} \mathrm{C}$, followed by 39 cycles of $94^{\circ} \mathrm{C}$ for 15 seconds, $58^{\circ} \mathrm{C}$ for 15 seconds, and $72^{\circ}$ for 15 seconds, with a final extension at $72^{\circ} \mathrm{C}$ for 5 minutes. The Resulting PCR were separated on $3 \%$ agarose gel electrophoresis and then visualized using RedSafe $^{\mathrm{TM}}$. The PCR products were either $242 \mathrm{bp}$ in the case of 3 tandem repeats (3R); or 214 bp in the case of 2 tandem repeats $(2 \mathrm{R})$.

Findings of the PCR-RFLP were validated by: 1) A negative control (run all PCR components except the DNA template) was run simultaneously with each PCR run; 2) Around $33 \%$ of all samples were repeated to confirm findings of the PCR-RFLP; 3 ) Randomly selected $10 \%$ of the samples were confirmed by direct DNA sequencing (AB 3730xl DNA Analyzer). The results were 100\% concordant.

\section{Haplotype analysis}

The interaction between genetic polymorphism at the two loci was assessed by evaluating the combinedgenotypes effects and haplotype analysis. We analyzed the haplotype frequencies of the two loci (TSER and 6bp ins/del) for lung cancer cases and compared them with those of controls. Haplotype frequencies were calculated using Multiallelic Interallelic Disequilibrium Analysis Software [(MIDAS $\left.{ }^{\circledR}\right)$, University of Southampton, Highfield, Southampton, UK (Gaunt et al., 2006) and linkage disequilibrium was represented by Lewontin's coefficient (D').

\section{Statistical analysis}

Data were coded and entered into SPSS software version 20 (IBM Corp, 2011). Data of categorical nature were summarized as counts and percentages. Data of continuous nature were summarized as mean \pm standard deviation. The relation between categorical vs. categorical variables were evaluated by Chi-square or Fisher exact test as appropriate. The strength of association was assessed by calculating odds ratio (OR) and $95 \%$ confidence interval (95\%CI) (Cochran, 1954). The relationship between categorical variable and continuous variable was evaluated by independent t-test. Hardy-Weinberg Equilibrium was assessed for genotypes and allele types. A p value $<0.05$ was considered statistically significant.

\section{Results}

DNA samples from three cases and one control failed to be amplified using TSER $2 R / 3 R$ primers. All cases and controls were appropriately matched with regards to gender, age, and smoking (Table 1). There were 16(18\%) lung cancer cases and $1(1.4 \%)$ control reported a family history of any type of cancer, which was associated with a significantly increased risk for lung cancer (OR: 16.5,95\% CI: 2.1-127.6, $\mathrm{p}<0.001)$. The allele frequencies of TSER $2 R$ and ts 3'-UTR 6bp ins were 0.41 and 0.39 , respectively, in the controls, while they were 0.4 and 0.48 , respectively, in the cases. The allelic distribution of the two loci of 
Thymidylate Synthase Polymorphisms and Risk of Lung Cancer among the Jordanian Population: a Case Control Study

interest were in Hardy-Weinberg equilibrium $(\mathrm{p}>0.05)$. The genotypic frequencies of the ts polymorphisms in cases and controls are demonstrated in table 2 .

Interaction of age, smoking status, and gender with the influence of ts genotypes on lung cancer incidence were studied. There was no statistically significant difference in the ts 3'-UTR $6 \mathrm{bp} \mathrm{del/ins} \mathrm{genotypes} \mathrm{distribution}$ between genders among cases and controls [( LC: Men: $15 \mathrm{ins} / \mathrm{ins}, 40 \mathrm{ins} / \mathrm{del}, 18 \mathrm{del} / \mathrm{del}$; Women: 3 ins/ins, 4 ins/ del, $4 \mathrm{del} / \mathrm{del}, \mathrm{p}=0.52)$; (Controls: Men: 7ins/ins, 35 ins/ del, $21 \mathrm{del} / \mathrm{del}$; Women: 0 ins/ins, $6 \mathrm{ins} / \mathrm{del}, 2 \mathrm{del} / \mathrm{del}$, $\mathrm{p}=0.47)]$. Similar results were observed when TSER $2 R / 3 R$ genotypes distribution between genders among cases and control were studied [(LC: Men: 14 2R/2R, 31 2R/3R, 25 3R/3R; Women:1 2R/2R, 3 2R/3R, 7 3R/3R, $p=0.21$ ); (Controls: Men:10 2R/2R, 27 2R/3R,25 3R/3R; Women: 4 $2 \mathrm{R} / 2 \mathrm{R}, 2 \mathrm{R} / 3 \mathrm{R}, 23 \mathrm{R} / 3 \mathrm{R}, \mathrm{p}=0.08$ )]. When gender is taken into consideration, the distribution of either polymorphs was similar among cases and controls.

The age can modify the influence of ts 3 '-UTR $6 \mathrm{bp}$ $\mathrm{del} /$ ins genotype on lung cancer susceptibility where individuals who were less than or equal 57 years carrying and ins/ins genotype were 4.6 times more susceptible to lung cancer $\left[\mathrm{OR}_{<57 \text { vs }>57 y \text { years }}: 4.6\right.$ (95\% CI: 0.93-22.46), $\mathrm{p}=0.059)$ ]. The age cut point was decided to be 57 based on the median age of the study population. However different age cut points were used to assess the robustness of the previous findings and the results were consistent; younger individuals bearing $6 \mathrm{bp}$ ins/ins genotype were always at increased risk for lung cancer development at higher age cut point $\left[\mathrm{OR}_{<60 \text { vs }>60 \text { years }}: 5.4(95 \% \mathrm{CI}: 1.44-\right.$ $20.13), \mathrm{p}=0.007)$ and lower age cut point $\left[\mathrm{OR}_{<52 \text { vs }>52 \text { years }}\right.$ : 9.6 (95\%CI: 1.11-8-3.40), $\mathrm{p}=0.017)]$. On the other hand, the interaction of age on the TSER $2 R / 3 R$ genotypes influence on lung cancer susceptibility revealed nonsignificant findings.

The interaction between ex-smoking and ts genotypes influence on lung cancer susceptibility was statistically significant only in ex-smokers carrying ins/ins genotype [Ex-smoker: ins/ins OR: 5.0(95\%CI: 0.87-28.86), $\mathrm{p}=0.028$, but it was complicated by small sample size, which may render the results unreliable. In an attempt to increase the sample size, "Smoking and ex-smoking" groups were combined and the analysis were repeated that revealed non-significant $\mathrm{p}$ value in all groups across different genotypes except for del/del genotype; where smokers or ex-smokers carrying del/del genotype are 3.3 times more protected against lung cancer development compared to those with ins/ins genotype [OR: 0.3(95\%CI: $0.10-0.94), p=0.04]$. Similar non-significant findings were observed when the combined effects of smoking and age on the influence of ts genotypes on lung cancer incidence were studied. Though $\mathrm{p}$ value was close to significant in those who are "smokers or ex-smokers and less than 57 years" with ins/ins genotype [OR: 4.0(95\%CI: 0.8020.41), $\mathrm{p}=0.07]$.

There were four haplotypes derived from the observed TYMS genotypes; 3R_del, 3R_ins, 2R_del and 2R_ins. The most frequent haplotype was 3R_del (cases: $31 \%$; controls: $43 \%$ while the rare haplotype was $2 \mathrm{R}$ ins (cases: 19\%; controls: $22 \%$ ). There was a significant difference
Table 1. Demographics of Lung Cancer Cases and Apparently Healthy Controls

\begin{tabular}{lcccc}
\hline \multirow{4}{*}{ Parameters } & \multicolumn{3}{c}{$\mathrm{N}(\%)$ or Mean \pm SD } & \\
\cline { 2 - 5 } & $\begin{array}{c}\text { Cases } \\
(\mathrm{n}=84)\end{array}$ & $\begin{array}{c}\text { Control } \\
(\mathrm{n}=71)\end{array}$ & $\begin{array}{c}\text { Total } \\
(\mathrm{n}=155)\end{array}$ & $\mathrm{p}$ \\
\hline Male Gender & $73(86.9 \%)$ & $63(88.7 \%)$ & $136(87.7 \%)$ & 0.5961 \\
Age (Years) & $57.1 \pm 9.6$ & $56.9 \pm 9.3$ & $57.0 \pm 9.4$ & 0.8722 \\
Smoker & $68(81.0 \%)$ & $56(78 \%)$ & $124(80 \%)$ & 0.9651 \\
\hline
\end{tabular}

Table 2. Genotypes and Allele Types of the $6 \mathrm{bp}$ Polymorphism and the TSERs among Lung Cancer Cases and their Matched Controls

\begin{tabular}{lrrll}
\hline Gene/Allele type Cases & $\begin{array}{r}\text { Controls } \\
(\mathrm{n}=84)\end{array}$ & OR ( $95 \%$ CI $)$ & p value \\
\hline 6bp ins/del & & & & \\
ins/ins & $18(21.4)$ & $7(9.9)$ & 1 (reference) & \\
ins/del & $44(52.4)$ & $41(57.7)$ & $0.42(0.16-1.10)$ & 0.07 \\
del/del & $22(26.2)$ & $23(32.4)$ & $0.37(0.13-1.06)$ & 0.06 \\
ins/ins & $18(21.4)$ & $7(9.9)$ & $2.5(0.98-6.37)$ & 0.051 \\
Others & $66(78.6)$ & $64(90.1)$ & & \\
insertion & $80(47.6)$ & $55(38.7)$ & $1.4(0.9-2.3)$ & 0.12 \\
deletion & $88(52.4)$ & $87(61.3)$ & & \\
TSER & & & & \\
3R/3R & $32(39.5)$ & $27(38.6)$ & $1($ reference $)$ & \\
2R/3R & $34(42.0)$ & $29(41.4)$ & $0.99(0.49-2.01)$ & 0.97 \\
2R/2R & $15(18.5)$ & $14(20.0)$ & $0.90(0.37-2.20)$ & 0.83 \\
3R/3R & $32(39.5)$ & $27(38.6)$ & $0.99(0.5-1.9)$ & 0.97 \\
Others & $49(60.5)$ & $41(61.4)$ & & \\
3R & $98(60.5)$ & $83(59.3)$ & $1.05(0.7-1.7)$ & 0.8 \\
2R & $64(39.5)$ & $57(40.7)$ & & \\
\hline
\end{tabular}

Ins: 6bp insertion; del: 6bp deletion; 2R: two TSERs, 3R: three TSERs, OR: odds ratio, CI: confidence interval

Table 3. Haplotype Frequencies of TS among Lung Cancer Patients and Controls

\begin{tabular}{lcclc}
\hline Haplotype & $\begin{array}{c}\text { Cases } \\
\text { Count (\%) }\end{array}$ & $\begin{array}{c}\text { Control } \\
\text { Count }(\%)\end{array}$ & P & OR (95\% CI) \\
\hline 3R_Del & $51(31 \%)$ & $60(43 \%)$ & 0.034 & $0.6(0.38-0.96)$ \\
3R_Ins & $46(28 \%)$ & $23(16 \%)$ & 0.016 & $2.0(1.13-3.48)$ \\
2R_Del & $36(22 \%)$ & $26(19 \%)$ & 0.47 & $1.2(0.70-2.17)$ \\
2R_Ins & $31(19 \%)$ & $31(22 \%)$ & 0.48 & $0.82(0.47-1.43)$ \\
\hline
\end{tabular}

Count represents the number of chromosomes. Ins: 6bp insertion; del: 6bp deletion; 2R: two TSERs, 3R: three TSERs, OR: odds ratio, CI: confidence interval

in the distribution of haplotypes between the cases and controls $(p=0.043)$. Our findings indicated that weak linkage disequilibrium existed between the two loci of interest as measured by Lewontin's coefficient (LC: D' $=0.03, r^{2}: 0.001, p=0.8$; Controls: $D^{\prime}=0.29, r^{2}: 0.08$, $\mathrm{p}=0.02)$. Carriers of the haplotype $3 \mathrm{R}$ insertion were 2 times more likely to have lung cancer, while the carriers of the 3R_deletion were 1.67 times more likely to protect from lung cancer (table 3).

\section{Discussion}

Smoking has been called the chief avoidable cause of lung cancer. It is now regarded as the most important public health issue, more than $50 \%$ of Jordanians are considered to be regular smokers despite the wide-spread 
knowledge among the Jordanian population that smoking is the main risk factor for lung and heart diseases (AlMatubsi et al., 2011). Other risk factors for lung cancer development are; second-hand smoke, asbestos, polycyclic aromatic hydrocarbons, radiation, pulmonary fibrosis and HIV infection, genetic and dietary factors (Alberg and Samet, 2003; Winkler-Heil et al., 2015). Reduced DNA repair capacity for getting rid of tobacco-induced adducts provides some evidence of inter individuals variation in susceptibility to lung cancer, as less than $20 \%$ of lifetime smokers develop lung cancer (Shi et al., 2005; Wang et al., 2013a).

Several studies were conducted to evaluate the effect of genetic polymorphisms involved in folate and one carbon unit metabolism pathway including; MTHFR, ts, and SLC19A1 and cancer incidence (Suzuki et al., 2007; Gao et al., 2012; Hu et al., 2012; Lu et al., 2012; Yang et al., 2012; Dajani et al., 2013; Galbiatti et al., 2013; Morita et al., 2013; Yousef et al., 2013; Rai, 2014; Al-Motassem et al., 2015; Awwad et al., 2015; Wang et al., 2015).

After comprehensive PubMed search was done utilizing lung cancer, polymorphism and thymidylate synthase as key words, only two articles were found with regard to the influence of ts genetic polymorphism on lung cancer incidence; one among Japanese and the second among non-Hispanic White (Shi et al., 2005; Suzuki et al., 2007).

Variability in 6 bp del/ins polymorphism in ts $3^{\prime}$ UTR has emerged as a possible risk factor for lung cancer in non-Hispanic white population, where individuals carrying ins/ins genotypes were at significantly increased risk of lung cancer (OR 1.52, 95\% CI (1.12-2.06)). ts3'UTR, but not the TSER variant was associated with the risk of lung cancer (Shi et al., 2005). Another study did not find any association between ts polymorphisms and cancer risk in general after in the cancer type and ethnic subgroup-stratification analyses had been done (Tang et al., 2012). TSER $2 R / 2 \mathrm{R}$ genotype carriers had a high odds ratio in gastric cancer and for the intestinal type, but was not statistically significant $(\mathrm{OR}=2.31,95 \% \mathrm{CI}=0.94-5.65$; and $\mathrm{OR}=2.53,95 \% \mathrm{CI}=0.98-6.54$, respectively) (Yim et al., 2010). Our study indicated that individuals who were less than or equal 57 years carrying ins/ins genotype were 4.6 times more susceptible to lung cancer, and the risk can be modified by smoking status as smokers and ex-smokers bearing ins/ins polymorphism were at increased risk for lung cancer among Jordanians.

Many studies on susceptibility to cancer or resistance to chemotherapy based on ts polymorphisms had been conducted based on the assumption that $3 \mathrm{R}$ allele as well as $6 \mathrm{bp}$ insertion allele are associated with increased ts expression (Yousef et al., 2001; Jung et al., 2013; Morita et al., 2013; Sulzyc-Bielicka et al., 2013; Wang et al., 2013b; Baroudi et al., 2014; Jang et al., 2014). The 6bp insertion allele had approximately 50\% higher mRNA expression than did 6bp deletion allele (Mandola et al., 2004). ts mRNA levels in tumor tissue were 3-fold lower among subjects that are homozygotes for $2 \mathrm{R} / 2 \mathrm{R}$ tandem repeat polymorphism in TSER than among subjects that are 3R/3R homozygotes (Pullarkat et al., 2001). Thus 3R/3R homozygous genotype had been associated with increased levels of ts protein expression and higher absolute enzyme activity (Shi et al., 2005).

It had been reported that ts $6 \mathrm{bp} \mathrm{del/del} \mathrm{genotype} \mathrm{put}$ people at higher risk of lung cancer, as any reduction in ts enzymatic activity resulting in increased uracil mis-incorporation in DNA and an increase in doublestrand breaks, complicated by reduced folate intake, and smoking. Impaired DNA repair capacity (reduced ts protein expression) for getting rid of tobacco-induced DNA adducts can further increase the risk of lung cancer development (Spitz et al., 2003). This case control study indicated that individuals with ins/ins polymorphism are at higher risk for lung cancer. The conflicting findings regarding ts genetic polymorphism and lung cancer incidence can be understood within the context of a number of findings: the linkage disequilibrium between ts 6bp in 3 UTR and ts tandem repeat in 5 UTR, and the integral role of ts in DNA repair especially among smokers. Other genetic polymorphisms among ts and other genes involved in folate metabolism may contribute to risk of lung cancer.

In this study, weak linkage disequilibrium between the two loci of interest existed, despite that fact that there was significant difference in the distribution of haplotypes between the cases and controls $p=0.04$. This may partly interpreted as the physical distance between the two loci is long (5 UTR and 3 UTR). Our findings showed inconsistency with Chinese breast cancer study results where TSER locus was partially in $\mathrm{LD}$ with the ts 3'-UTR del6 locus ( $\mathrm{D}^{\prime}=0.584 ; \mathrm{R}^{2}=0.186, \mathrm{P}<0.001$ ) and no significant difference in the distribution of haplotypes among cases and controls (Zhai et al., 2006). Similar results were demonstrated in a study conducted in Italy to predict response to 5-fluorouracil-based chemotherapy in liver only metastatic colorectal cancer based on ts polymorphisms where ts 5'-UTR and ts 3'-UTR loci showed mild LD $\left(r^{2}=0.17\right)$ (Graziano et al., 2008). Hence, studying the association between ts haplotypes and lung cancer incidence is less worthwhile than studying the ts3 UTR del/ins polymorphism in defining individuals at higher risk of lung cancer.

The study findings are limited by: 1 . Small sample size. 2. No available reliable informative methods of detecting dietary intake of folate, vitamin B6, and vitamin B12.

In conclusion, the findings of the current study suggest that genetic polymorphism of ts at 3 UTR and its haplotype analysis modulates the risk of lung cancer in the Jordanian population. Polymorphism of ts at 3 'UTR is more informative than TSER polymorphism in predicting Jordanian population at increased risk of lung cancer.

\section{Acknowledgements}

This study was supported by unconditional grant from the Deanship of Scientific Research (University of Jordan, Jordan). The study sponsors had no part in the study design, data collection, data analysis, data interpretations or in the writing of the manuscript. The sponsors had no role in the decision to submit the paper for publication. 


\section{References}

Al-Matubsi HY, Kanaan RA, Hamdan F, et al (2011). Smoking practices in Jordanian people and their impact on semen quality and hormonal levels among adult men. Central European Journal Of Public Health, 19, 54-9.

Al-Motassem Y, Shomaf M, Said I, et al (2015). Allele and Genotype Frequencies of the Polymorphic Methylenetetrahydrofolate Reductase and Lung Cancer in ther Jordanian Population: a Case Control Study. Asian Pac J Cancer Prev, 16, 3101-9.

Alberg AJ, Samet JM (2003). Epidemiology of lung cancer. Chest, 123, 21-49.

Awwad N, Yousef AM, Abuhaliema A, et al (2015). Relationship between Genetic Polymorphisms in MTHFR (C677T, A1298C and their Haplotypes) and the Incidence Of Breast Cancer among Jordanian Females - Case-Control Study. Asian Pac J Cancer Prev, 16, 5007-11.

Baroudi O, Baroudi T, Omrane I, et al (2014). Thymidylate synthase polymorphism in sporadic colorectal and gastric cancer in Tunisian population: a predictive role in 5-fluorouracil based chemotherapy treatment. Med Oncol, 31, 825 .

Cochran G (1954). Some Methods for Strengthening the Common $\chi$ Tests. Biometrics, 10, 417-51.

Dajani R, Fathallah R, Arafat A, et al (2013). Prevalence of MTHFR C677T single nucleotide polymorphism in genetically isolated populations in Jordan. Biochem Genet, 51, 780-8.

Galbiatti AL, Castro R, Caldas HC, et al (2013). Alterations in the expression pattern of MTHFR, DHFR, TYMS, and SLC19A1 genes after treatment of laryngeal cancer cells with high and low doses of methotrexate. Tumour Biol, 34, 3765-71.

Gao CM, Ding JH, Li SP, et al (2012). Polymorphisms in the thymidylate synthase gene and risk of colorectal cancer. Asian Pac J Cancer Prev, 13, 4087-91.

Gaunt TR, Rodriguez S, Zapata C, et al (2006). MIDAS: software for analysis and visualisation of interallelic disequilibrium between multiallelic markers. BMC Bioinformatics, 7, 227.

Graziano F, Ruzzo A, Loupakis F, et al (2008). Liver-only metastatic colorectal cancer patients and thymidylate synthase polymorphisms for predicting response to 5-fluorouracil-based chemotherapy. Br J Cancer, 99, 716-21.

Hensing T, Chawla A, Batra R, et al (2014). A personalized treatment for lung cancer: molecular pathways, targeted therapies, and genomic characterization. Adv Exp Med Biol, 799, 85-117.

Horie N, Aiba H, Oguro K, et al (1995). Functional analysis and DNA polymorphism of the tandemly repeated sequences in the 5'-terminal regulatory region of the human gene for thymidylate synthase. Cell Struct Funct, 20, 191-7.

Horn L, Pao W, Johnson DH (2012). Chapter 89. Neoplasms of the Lung. In 'Harrison's Principles of Internal Medicine, 18e', Eds The McGraw-Hill Companies, New York, NY,

Hu HB, Kuang L, Zeng XM, et al (2012). Predictive value of thymidylate synthase expression in gastric cancer: a systematic review with meta-analysis. Asian Pac J Cancer Prev, 13, 261-7.

Islami F, Ward EM, Jacobs EJ, et al (2015). Potentially preventable premature lung cancer deaths in the USA if overall population rates were reduced to those of educated whites in lower-risk states. Cancer Causes Control, 26, 409-18.

Jang MJ, Kim JW, Jeon YJ, et al (2014). Polymorphisms of folate metabolism-related genes and survival of patients with colorectal cancer in the Korean population. Gene,
533, 558-64.

Jung M, Lee CH, Park HS, et al (2013). Pharmacogenomic assessment of outcomes of pemetrexed-treated patients with adenocarcinoma of the lung. Yonsei Med J, 54, 854-64.

Kumagai K, Hiyama K, Oyama T, et al (2003). Polymorphisms in the thymidylate synthase and methylenetetrahydrofolate reductase genes and sensitivity to the low-dose methotrexate therapy in patients with rheumatoid arthritis. Int J Mol Med, 11, 593-600.

Longley DB, Harkin DP, Johnston PG (2003). 5-fluorouracil: mechanisms of action and clinical strategies. Nat Rev Cancer, 3, 330-8.

Lu M, Sun L, Yang J, et al (2012). 3R variant of thymidylate synthase 5'-untranslated enhanced region contributes to colorectal cancer risk: a meta-analysis. Asian Pac J Cancer Prev, 13, 2605-10.

Lv YT, Du PJ, Wang QY, et al (2013). A novel approach to cloning and expression of human thymidylate synthase. Asian Pac J Cancer Prev, 14, 7523-7.

Mandola MV, Stoehlmacher J, Zhang W, et al (2004). A 6 bp polymorphism in the thymidylate synthase gene causes message instability and is associated with decreased intratumoral ts mRNA levels. Pharmacogenetics, 14, 319-27.

Morita M, Yin G, Yoshimitsu S, et al (2013). Folate-related nutrients, genetic polymorphisms, and colorectal cancer risk: the fukuoka colorectal cancer study. Asian Pac J Cancer Prev, 14, 6249-56.

Pullarkat ST, Stoehlmacher J, Ghaderi V, et al (2001). Thymidylate synthase gene polymorphism determines response and toxicity of 5-FU chemotherapy. Pharmacogenomics $J, \mathbf{1}$, 65-70.

Rai V (2014). Folate pathway gene MTHFR C677T polymorphism and risk of lung cancer in Asian populations. Asian Pac J Cancer Prev, 15, 9259-64.

Shi Q, Zhang Z, Neumann AS, et al (2005). Case-control analysis of thymidylate synthase polymorphisms and risk of lung cancer. Carcinogenesis, 26, 649-56.

Spitz MR, Wei Q, Dong Q, et al (2003). Genetic susceptibility to lung cancer: the role of DNA damage and repair. Cancer Epidemiol Biomarkers Prev, 12, 689-98.

Sulzyc-Bielicka V, Bielicki D, Binczak-Kuleta A, et al (2013). Thymidylate synthase gene polymorphism and survival of colorectal cancer patients receiving adjuvant 5-fluorouracil. Genet Test Mol Biomarkers, 17, 799-806.

Suzuki T, Matsuo K, Hiraki A, et al (2007). Impact of onecarbon metabolism-related gene polymorphisms on risk of lung cancer in Japan: a case control study. Carcinogenesis, 28, 1718-25.

Tang J, Wang PP, Zhuang YY, et al (2012). Thymidylate synthase genetic polymorphisms and cancer risk: a meta-analysis of 37 case-control studies. Chin Med J (Engl), 125, 2582-8.

Tarawneh M, Nimri O, Arkoob K, et al 2010. cancer incidence in Jordan-2010 [Online]. Amman, Jordan: Ministry of Health. Available: www.moh.jov.jo/documents/annual $\% 20$ incidence\%20of\%20cancer\%20in\%20Jordan@202010.pdf [Accessed July,2015 2015].

Ulrich CM, Bigler J, Bostick R, et al (2002). Thymidylate synthase promoter polymorphism, interaction with folate intake, and risk of colorectal adenomas. Cancer Res, $\mathbf{6 2}$, 3361-4.

Wang LE, Gorlova OY, Ying J, et al (2013a). Genome-wide association study reveals novel genetic determinants of DNA repair capacity in lung cancer. Cancer Res, 73, 256-64.

Wang X, Yue K, Hao L (2015). Meta-analysis of methylenetetrahydrofolate reductase polymorphism and lung cancer risk in Chinese. Int J Clin Exp Med, 8, 1521-5.

Wang YC, Xue HP, Wang ZH, et al (2013b). An integrated 
Wiam Al Qasem et al

analysis of the association between ts gene polymorphisms and clinical outcome in gastric and colorectal cancer patients treated with 5-FU-based regimens. Mol Biol Rep, 40, 4637-44.

Winkler-Heil R, Hofmann W, Hussain M, et al (2015). Analyses of local dose distributions in the lungs for the determination of risk apportionment factors. Radiat Prot Dosimetry.

Wu GQ, Liu NN, Xue XL, et al (2014). Multiplex real-time PCR for RRM1, XRCC1, TUBB3 and ts mRNA for prediction of response of non-small cell lung cancer to chemoradiotherapy. Asian Pac J Cancer Prev, 15, 4153-8.

Wu X, Zhao H, Suk R, et al (2004). Genetic susceptibility to tobacco-related cancer. Oncogene, 23, 6500-23.

Yang Z, Liu HX, Zhang XF (2012). 2R of thymidylate synthase 5 '-untranslated enhanced region contributes to gastric cancer risk: a meta-analysis. Asian Pac J Cancer Prev, 13, 1923-7.

Yim DJ, Kim OJ, An HJ, et al (2010). Polymorphisms of thymidylate synthase gene 5'- and 3'-untranslated region and risk of gastric cancer in Koreans. Anticancer Res, 30, 2325-30.

Yousef A-M, Shomaf M, Berger S, et al (2013). Allele and genotype frequencies of the polymorphic methylenetetrahydrofolate reductase and colorectal cancer among Jordanian population. Asian Pacific Journal of Cancer Prevention, 14, 4559-65.

Yousef AM, Davis RA, Sticca RP, et al (2001). Structural analysis of cDNA encoding thymidylate synthase in hepatic metastases of human colorectal tumors. Int $J$ Colorectal Dis, 16, 318-25.

Zhai X, Gao J, Hu Z, et al (2006). Polymorphisms in thymidylate synthase gene and susceptibility to breast cancer in a Chinese population: a case-control analysis. BMC Cancer, $\mathbf{6}, 138$. 